\section{Emergency endocrine cases: glucose gone wrong \\ 1. Pragmatic approach to managing a DKA patient}

\section{Robert Shiel}

When the supply of glucose is compromised, ketones (acetone, beta-hydroxybutyrate and acetoacetate) are produced in the liver from fat mobilized from peripheral stores as an alternative energy substrate. Although normally produced at low levels within the body, ketone production increases in the presence of an absolute or relative insulin deficiency; a process intensified by insulin antagonist hormones, particularly glucagon. Therefore, in diabetic animals, ketoacidosis is the result of a reduced insulin:glucagon ratio and not persistent hyperglycaemia. There is no negative feedback mechanism for the production of ketones, and acidaemia occurs when ketone production exceeds the body's energy requirement and buffering capacity. Acidaemia can lead to adverse effects including vomiting, dullness and anorexia, which in turn exacerbate electrolyte and water disturbances and worsen glycaemic control. Furthermore, diabetic ketoacidosis (DKA) most commonly develops in the presence of concurrent illnesses such as infection or pancreatitis, which can further complicate the clinical picture. As a result, DKA can be life threatening.

Diagnosis is straightforward, based upon documentation of metabolic acidosis and ketosis in a diabetic patient. Additional tests are recommended to characterize the severity of electrolyte and other derangements, and to explore potential concurrent diseases.

There are three goals of therapy: to decrease ketone production, manage electrolyte and fluid derangements, and treat any precipitating concurrent disease. Since hyperglycaemia is not the cause of ketoacidosis, the aim of therapy need only be to switch off ketone production rather than attempting to normalize blood glucose. Theoretically at least, this should be achievable with relatively low doses of insulin. Low-dose regimens also have the benefit of not reducing blood glucose too quickly which could lead to cerebral oedema and neurological signs.

Fluid therapy is used to correct water and electrolyte disturbances. Normal saline is appropriate in the early stages of therapy but dextrose saline is the fluid of choice once insulin therapy starts to reduce blood glucose concentrations. Intravenous (or intramuscular followed by subcutaneous) soluble insulin protocols are used to reduce ketone production, and to decrease glucose concentrations to a goal of $10-15 \mathrm{mmol} / \mathrm{l}$. Potassium concentrations are often depleted in DKA patients, and this is exacerbated when insulin therapy is commenced. Hypokalaemia typically develops within hours of starting treatment. Affected animals are weak and lethargic, which may be confused with signs of DKA. It is usually necessary to add potassium to intravenous fluids to prevent severe hypokalaemia. However, accurate supplementation is extremely difficult without the ability to measure potassium on-site. Intravenous phosphate supplementation may also be required, particularly after 12-24 hours of therapy. Once dehydration is corrected, vomiting has ceased and appetite returned, standard diabetes-management protocols can be commenced or resumed. Underlying diseases should be treated as appropriate.

\section{KEY LEARNING OBJECTIVES}

- Identify factors behind development of diabetic ketoacidosis to understand treatment goals better

- Have the knowledge to manage affected dogs and cats, adjusting treatment strategies in response to changing clinical parameters

- Be aware of possible complicating factors that can arise in response to therapy

\section{MULTIPLE CHOICE QUESTIONS}

1. Treatment of DKA is commenced in a cat and there is a good clinical response. However, urine ketone measurements increase following initial treatment. What is the significance of this finding?

(A) It probably reflects metabolism of beta-hydroxybutyrate to acetoacetate and acetone and is not of concern

(B) It probably suggests worsening of the severity of acidaemia, which should be addressed with more aggressive therapy

(C) Increased urinary ketone secretion is probably due to the administration of intravenous fluids and it is therefore not of concern

(D) It probably reflects production of ketones in the urine due to concurrent urinary tract infection, which should be treated

2. What is the main clinical sign of hypophosphataemia in a cat undergoing therapy for DKA?
(A) Seizures
(B) Muscle weakness
(C) Vomiting
(D) Haemolytic anaemia

3. Which of the following endocrine diseases is LEAST likely to be associated with DKA?
(A) Hyperthyroidism
(B) Hyperadrenocorticism
(C) Hypothyroidism
(D) Hypoadrenocorticism 